\title{
MEROMORPHIC SOLUTIONS OF NON-LINEAR DIFFERENTIAL-DIFFERENCE EQUATIONS
}

\section{GUOZHEN REN and LINGYUN GAO}

Department of Mathematics

Jinan University

Guangzhou, Guangdong 510632

P. R. China

e-mail: 1104832904@qq.com

\begin{abstract}
Using Nevanlinna theory of the value distribution of meromorphic functions and complex differential equations theory, we investigate the properties of one type of non-linear differential-difference equation with rational solution and the existence of transcendental meromorphic solutions of another type of equation, and obtain some results.
\end{abstract}

\section{Introduction and Main Results}

We use the standard symbols and fundamental results of Nevanlinna theory [1, 14]. A function $a(z)(\equiv 0, \infty)$ is called a small function with respect to $w(z)$, if $T(r, a)=S(r, w)$, where $S(r, w)$ denotes any quantity satisfying $\quad S(r, w)=o(T(r, w))$ with $r \rightarrow \infty$ outside of a possible

2010 Mathematics Subject Classification: 30D35, 39A12.

Keywords and phrases: differential-difference equation, rational solution, meromorphic functions.

This project is supported by NSF of P. R. China (No.11271161).

Received March 11, 2019; Revised May 8, 2019

(C) 2019 Scientific Advances Publishers 
exceptional set of finite logarithmic measure. For a meromorphic function $w(z)$, the order and the hyper-order of $w$ is respectively defined by

$$
\begin{gathered}
\rho(w)=\limsup _{r \rightarrow \infty} \frac{\log T(r, w)}{\log r}, \\
\sigma(w)=\limsup _{r \rightarrow \infty} \frac{\log \log T(r, w)}{\log r} .
\end{gathered}
$$

Moreover, we use the notation $\operatorname{deg}_{w}(P)$ for the degree of $P(z, w(z))$ with respect to $w$.

Recently, some papers focused on complex difference equations of Nevanlinna's theory and difference analogue. Many authors ([2-9]) have discussed the existence and the growth of meromorphic solutions of difference equations and obtained many results.

In 2017, Halburd and Korhonen [10] investigated the properties of meromorphic solutions of non-linear differential-difference equation

$$
w(z+1)-w(z-1)+a(z) \frac{w^{\prime}(z)}{w(z)}=R(z, w(z))=\frac{P(z, w(z))}{Q(z, w(z))},
$$

where $a(z)$ is a rational function, $P(z, w(z))$ is a polynomial in $w$ having rational coefficients in $z, Q(z, w(z))$ is a monic polynomial in $w, P(z, w)$ and $Q(z, w)$ are co-prime.

They obtained the following result:

Theorem A ([10]). Let $w(z)$ be a non-rational solution of (1). If $\sigma(w)<1$, then

$$
\operatorname{deg}_{w}(P)=\operatorname{deg}_{w}(Q)+1 \leq 3
$$

or the degree of $R(z, w)$ as a rational function in $w$ is either 0 or 1.

In 2018, Liu et al. [11] considered the following non-linear differential-difference equation:

$$
c(z) w(z+1)+a(z) \frac{w^{\prime}(z)}{w(z)}=R(z, w(z))=\frac{P(z, w(z))}{Q(z, w(z))},
$$


where $R(z, w(z))$ is rational function in $w(z), a(z)$ and $c(z)$ are non-zero rational functions. $P(z, w(z))$ and $Q(z, w(z))$ are defined in (1). They have considered the case that (2) admits a rational solution where all the coefficients of (2) are constants. A result was obtained as follows.

Theorem B ([11]). Let $w(z)=\frac{M(z)}{N(z)}$ be a non-constant rational solution of (2), $M(z)$ and $N(z)$ be polynomials as follows:

$$
M(z)=a_{m} z^{m}+\cdots+a_{1} z+a_{0}, \quad N(z)=b_{n} z^{n}+\cdots+b_{1} z+b_{0},
$$

and let $h:=P(z, 0), g:=P^{\prime}(z, 0), e:=Q(z, 0)$ in $(2)$, we have

(1) if $\operatorname{deg}_{w}(P)=0$, then $m=n$;

(2) if $\operatorname{deg}_{w}(P)=3, \operatorname{deg}_{w}(Q)=2$, then $m \geq n$ except that $n=m+1$ provided that $h=0, e \neq 0$ and $g \neq 0$.

In this paper, we mainly consider the existence problem of meromorphic solutions of the Equations (3) and (4) as follows:

$$
\begin{gathered}
c(z) w(z+1)+a(z) \frac{w^{\prime}(z)}{w(z)}+b(z) \frac{w^{\prime \prime}(z)}{w(z)}=R(z, w(z))=\frac{P(z, w(z))}{Q(z, w(z))}, \\
c(z) w(z+1)+a(z) \frac{w^{\prime}(z)}{w(z)}+b(z) \frac{w^{\prime \prime}(z)}{w(z)}=d(z),
\end{gathered}
$$

where $a(z), b(z), c(z), d(z)$ are non-zero rational functions, $P(z, w(z))=$ $\sum_{i=0}^{l} c_{i} w^{l-i}(z)$ is a polynomial in $w, Q(z, w(z))=\sum_{j=0}^{l} c_{j} w^{l-j}(z)$ is a monic polynomial in $w$, and they have no common roots, $\left\{c_{i}(z)\right\},\left\{d_{j}(z)\right\}$ are rational functions in $z$.

Moreover, we mainly consider the case that all the coefficients of (3) are constants.

Let $c_{l}=P(z, 0), c_{l-1}=P^{\prime}(z, 0), d_{l}=Q(z, 0) . d_{l}$ and $c_{l}$ can not vanish at the same time. We will prove 
Theorem 1. Let $w(z)=\frac{M(z)}{N(z)}$ be a non-constant rational solution of (3), $M(z)$ and $N(z)$ be polynomials as follows:

$$
M(z)=a_{m} z^{m}+\cdots+a_{1} z+a_{0}, \quad N(z)=b_{n} z^{n}+\cdots+b_{1} z+b_{0} .
$$

We have

(1) if $\operatorname{deg}_{w}(P)=0$, then $m=n$;

(2) if $\operatorname{deg}_{w}(P)=l, \operatorname{deg}_{w}(Q)=l-1(1 \leq l \leq 7)$, then $m \geq n$, particularly, if $c_{l}=0, c_{l-1} \neq 0$ and $d_{l} \neq 0$, then $n=m+1$.

Theorem 2. If $a(z) w^{\prime}(z)+b(z) w^{\prime \prime}(z) \neq 0$, then (4) has no transcendental entire solutions.

Theorem 3. Suppose that $w(z)$ is a transcendental meromorphic solution of the Equation (4), then $T(r, w)=N(r, w)+S(r, w)$.

\section{Some Lemmas}

We will use the following lemmas in our proofs of the above theorems.

Lemma 1 ([12]). Let $w$ be a meromorphic function and $R(z, w)$ be a rational function in $w$ and meromorphic in $z$. If all the coefficients of $R(z, w)$ are small compared to $w$, then

$$
T(z, R(z, w))=\operatorname{deg}_{w}(R) T(r, w)+S(r, w) .
$$

Lemma 2 ([13]). Let $T:[0, \infty) \rightarrow[0, \infty)$ be a non-decreasing continuous function and let $s \in(0, \infty)$. If the hyper-order of $T$ is strictly less than one, i.e.,

$$
\limsup _{r \rightarrow \infty} \frac{\log \log T(r)}{\log r}=\sigma<1
$$


and $\delta \in(0,1-\sigma)$, then

$$
T(r+s)=T(r)+o\left(\frac{T(r)}{r^{\delta}}\right),
$$

where $r$ runs to infinity outside of a set of finite logarithmic measure.

Lemma 3 ([13]). Let $w$ be a non-constant meromorphic function and $c \in C$. If $w$ is of finite order, then

$$
m\left(r, \frac{w(z+c)}{w(z)}\right)=O\left(\frac{\log r}{r} T(r, w)\right),
$$

for all $r$ outside of a set $E$ satisfying

$$
\limsup _{r \rightarrow \infty} \frac{\int_{E \bigcap[1, r) \frac{d t}{t}}}{\log r}=0,
$$

i.e., outside of a set $E$ of zero logarithmic density. If $\sigma(w)=\sigma<1$ and $\varepsilon>0$, then

$$
m\left(r, \frac{w(z+c)}{w(z)}\right)=o\left(\frac{T(r, w)}{r^{1-\sigma-\varepsilon}}\right)
$$

for all $r$ outside of a set of finite logarithmic measure.

\section{Proof of Theorem 1}

Taking Nevanlinna characteristic function of both sides of (3) and applying Lemma 1, we have

$$
\begin{aligned}
T\left(r, c(z) w(z+1)+a(z) \frac{w^{\prime}(z)}{w(z)}+b(z) \frac{w^{\prime \prime}(z)}{w(z)}\right) & =T(r, R(z, w)) \\
& =\operatorname{deg}_{w}(R) T(r, w(z))+O(\log r) .
\end{aligned}
$$


Using Lemmas 2 and 3, and using the logarithmic derivative lemma [12], it follows that

$$
\begin{aligned}
\operatorname{deg}_{w}(R) T(r, w(z)) \leq & T(r, w(z+1))+T\left(r, \frac{w^{\prime}(z)}{w(z)}\right)+T\left(r, \frac{w^{\prime \prime}(z)}{w(z)}\right)+O(\log r) \\
\leq & T(r, w)+N\left(r, \frac{w^{\prime}(z)}{w(z)}\right)+N\left(r, \frac{w^{\prime \prime}(z)}{w(z)}\right)+S(r, w) \\
\leq & T(r, w)+\left(\bar{N}(r, w(z))+\bar{N}\left(r, \frac{1}{w(z)}\right)\right) \\
& +2\left(\bar{N}(r, w(z))+\bar{N}\left(r, \frac{1}{w(z)}\right)\right)+S(r, w) \\
\leq & 7 T(r, w)+S(r, w) .
\end{aligned}
$$

Thus, we have

$$
\left(\operatorname{deg}_{w}(R)-7\right) T(r, w(z)) \leq S(r, w),
$$

which implies that $\operatorname{deg}_{w}(R)<7$, i.e.,

$$
\operatorname{deg}_{w}(P) \leq 7, \operatorname{deg}_{w}(Q) \leq 7 .
$$

We will discuss the following two cases.

Case 1: $\operatorname{deg}_{w}(P)=0$ and $\operatorname{deg}_{w}(Q)=l(l=0, \cdots, 7)$, we consider the following equation:

$$
c w(z+1)+a \frac{w^{\prime}(z)}{w(z)}+b \frac{w^{\prime \prime}(z)}{w(z)}=\frac{c_{0}}{\sum_{j=0}^{l} d_{j} w^{l-j}(z)},
$$

where $d_{j}(j=1, \cdots, l)$ are constants, $a, b, c, c_{0}, d_{0}=1$ are non-zero constants. Substituting $w(z)=\frac{M(z)}{N(z)}$ into (5), we have

$$
\begin{aligned}
& \frac{c M \bar{M} N^{2}+a M^{\prime} N^{2} \bar{N}-a M N N^{\prime} \bar{N}+b M^{\prime \prime} N^{2} \bar{N}-b M N N^{\prime \prime} \bar{N}-2 b M^{\prime} N N^{\prime} \bar{N}+2 b M\left(N^{\prime}\right)^{2} \bar{N}}{M N^{2} \bar{N}} \\
& =\frac{c_{0} N^{l}}{d_{0} M^{l}+d_{l} M^{l-1} N+d_{2} M^{l-2} N^{2}+\cdots+d_{j} M^{l-j} N^{j}+\cdots+d_{l} N^{l}} .
\end{aligned}
$$


According to the above equation, it follows that

$$
\begin{aligned}
& c \sum_{j=0}^{l} d_{j} M^{l-j+1} \bar{M} N^{j+2}+a \sum_{j=0}^{l} d_{j} M^{l-j} M^{\prime} N^{j+2} \bar{N} \\
& \quad-a \sum_{j=0}^{l} d_{j} M^{l-j+1} N^{j+1} N^{\prime} \bar{N}+b \sum_{j=0}^{l} d_{j} M^{l-j} M^{\prime \prime} N^{j+2} \bar{N} \\
& \quad-b \sum_{j=0}^{l} d_{j} M^{l-j+1} N^{j+1} N^{\prime \prime} \bar{N}-2 b \sum_{j=0}^{l} d_{j} M^{l-j} M^{\prime} N^{j+1} N^{\prime} \bar{N} \\
& \quad+2 b \sum_{j=0}^{l} d_{j} M^{l-j+l} N^{j}\left(N^{\prime}\right)^{2} \bar{N}=c_{0} M N^{l+2} \bar{N}
\end{aligned}
$$

Thus we see that the possible degrees of left-hand side of (6) are $(l-j+2) m+(j+2) n,(l-j+1) m+(j+3) n-1$ or $(l-j+1) m+(j+3) n$ $-2(j=0, \cdots, l)$. The coefficients of maximum degree terms are different, so there are no terms that can be cancelled. And we can see that the degree of right-hand side of $(6)$ is $m+(l+3) n$.

If $m \geq n+1$, we have the maximum degree of left-hand side of (6) is $(l+2) m+2 n$ and the degree of right-hand side of $(6)$ is $m+(l+3) n$, then $m=n$, a contradiction. If $m<n-1$, we have

$$
\begin{aligned}
& (l+2) m+2 n \leq(l+1) m+3 n-2<(l+1) m+3 n-1<(l+1) m+3 n \\
& \leq l m+4 n-2<l m+4 n-1<l m+4 n \leq \cdots \leq(l-j+1) m+(j+3) n \\
& \quad-2<(l-j+1) m+(j+3) n-1<(l-j+1) m+(j+3) n \leq \cdots \leq m \\
& \quad+(l+3) n-2<m+(l+3) n-1<m+(l+3) n .
\end{aligned}
$$

(i) If $d_{l} \neq 0$, then the degree of left-hand side of (6) is $m+(l+3) n-1$, since $c_{0} \neq 0$, the degree of right-hand side of (6) is $m+(l+3) n$, thus $m+(l+3) n-1=m+(l+3) n$, which is impossible. 
(ii) If $d_{l}=0$ and $c_{0} \neq 0$, in this case, the degree of right-hand side of (6) is $m+(l+3) n$, if $d_{l-1} \neq 0$, then the degree of left-hand side of (6) is $2 m+(l+2) n-1$, then $n=m-1$, which is a contradiction with the assumption $m<n-1$; if $d_{l-1}=0$ and $d_{l-2} \neq 0$, then the degree of lefthand side of (6) is $3 m+(l+1) n-1$, then $2 n=2 m-1$, a contradiction.

Similarly, if $d_{j}=0(j=1, \cdots, l)$, we have $l n=l m-1$ a contradiction again. If $m=n-1$, then

$$
\begin{aligned}
(l+1) m & +3 n-2<(l+2) m+2 n=(l+1) m+3 n-1=l m+4 n-2 \\
< & (l+1) m+3 n=l m+4 n-1=(l-1) m+5 n-2<\cdots=(l-j+1) m \\
& +(j+3) n-2<(l-j+2) m+(j+2) n=(l-j+1) m+(j+3) n-1 \\
= & (l-j) m+(j+4) n-2<(l-j+1) m+(j+3) n<\cdots=m+(l+3) n \\
& -2<2 m+(l+2) n=m+(l+3) n-1<m+(l+3) n .
\end{aligned}
$$

(i) If $d_{l} \neq 0$, then the degree of left-hand side of (6) is $m+(l+3) n-1$, since $c_{0} \neq 0$, the degree of right-hand side of (6) is $m+(l+3) n$, thus $m+(l+3) n-1=m+(l+3) n$, which is impossible.

(ii) If $d_{l}=0$ and $d_{l-1} \neq 0$, then the degree of left-hand side of (6) is $2 m+(l+2) n-1$, then $n=m-1$, which is a contradiction with the assumption $m=n-1$; if $d_{l-1}=0$ and $d_{l-2} \neq 0(j=l)$, then the degree of left-hand side of (6) is $3 m+(l+1) n-1$, then $2 n=2 m-1$, a contradiction.

Similarly, if $d_{j}=0(j=1, \cdots, l)$, we have $l n=l m-1$ a contradiction again. Anyway, $m \leq n-1$ is impossible.

Thus, we have $m=n$ if $\operatorname{deg}_{w}(P)=0$ and $\operatorname{deg}_{w}(Q)=l(l=0, \cdots, 7)$. 
Case 2: $\operatorname{deg}_{w}(P)=l$ and $\operatorname{deg}_{w}(Q)=l-1(l=1, \cdots, 7)$, we consider the following equation:

$$
c w(z+1)+a \frac{w^{\prime}(z)}{w(z)}+b \frac{w^{\prime \prime}(z)}{w(z)}=\frac{\sum_{i=0}^{l} c_{i} w^{l-i}(z)}{\sum_{j=1}^{l} d_{j} w^{l-j}(z)},
$$

where $c_{i}(i=0, \cdots, l), d_{j}(j=2, \cdots, l)$ are constants and $a, b, c, c_{0}, d_{1}=1$ are non-zero constants. Substituting $w(z)=\frac{M(z)}{N(z)}$ into (7), we have $\frac{c M \bar{M} N^{2}+a M^{\prime} N^{2} \bar{N}-a M N N^{\prime} \bar{N}+b M^{\prime \prime} N^{2} \bar{N}-b M N N^{\prime \prime} \bar{N}-2 b M^{\prime} N N^{\prime} \bar{N}+2 b M\left(N^{\prime}\right)^{2} \bar{N}}{M N^{2} \bar{N}}$ $=\frac{\sum_{i=0}^{l} c_{i} M^{l-i} N^{i}}{\sum_{j=1}^{l} d_{j} M^{l-j} N^{j}}$.

According to the above equation, it follows that

$$
\begin{aligned}
& c \sum_{j=1}^{l} d_{j} M^{l-j+1} \bar{M} N^{j+2}+a \sum_{j=1}^{l} d_{j} M^{l-j} M^{\prime} N^{j+2} \bar{N} \\
& \quad-a \sum_{j=1}^{l} d_{j} M^{l-j+1} N^{j+1} N^{\prime} \bar{N}+b \sum_{j=1}^{l} d_{j} M^{l-j} M^{\prime \prime} N^{j+2} \bar{N} \\
& \quad-b \sum_{j=1}^{l} d_{j} M^{l-j+1} N^{j+1} N^{\prime \prime} \bar{N}-2 b \sum_{j=1}^{l} d_{j} M^{l-j} M^{\prime} N^{j+1} N^{\prime} \bar{N} \\
& \quad+2 b \sum_{j=1}^{l} d_{j} M^{l-j+1} N^{j}\left(N^{\prime}\right)^{2} \bar{N}=\sum_{i=0}^{l} c_{i} M^{l-i+1} N^{i+2} \bar{N}
\end{aligned}
$$


Thus we see that the possible degrees of left-hand side of (8) are $(l-j+2) m+(j+2) n,(l-j+1) m+(j+3) n-1$ or $(l-j+1) m+(j+3) n-$ $2(j=1, \cdots, l)$ and the possible degrees of right-hand side of (8) are $(l-i+1) m+(i+3) n$, where $i=0, \cdots, l$.

If $m \geq n$, since $c, d_{l}$ are non-zero constants, the degree of both hand sides of (8) are equal, both are $(l+1) m+3 n$, this case may happen.

Next, we assume that $m<n$ in the following. We have $(l+1) m+3 n<l m+4 n<\cdots<(l-i+1) m+(i+3) n<\cdots<2 m+(l+2) n<$ $m+(l+3) n$.

(i) If $d_{l} \neq 0$, then the degree of left-hand side of (8) is at most $m+(l+3) n-1$, if $c_{l} \neq 0$, the degree of right-hand side of (8) is at most $m+(l+3) n$, thus $m+(l+3) n-1=m+(l+3) n$, which is impossible; if $c_{l}=0$ and $c_{l-1} \neq 0$, the degree of right-hand side of (8) is $2 m+(l+2) n$, then we have

$$
m+(l+3) n-1=2 m+(l+2) n .
$$

So $n=m+1$. If $c_{l}=c_{l-1}=0$, and $c_{l-2} \neq 0$, by comparing the degrees of the two hand sides of (8), it yields $2 n=2 m+1$, which is a contradiction.

Similarly, if $c_{i}=0(i=1, \cdots, l)$ and $c_{0} \neq 0$, then we have $\ln =\operatorname{lm}+1$, a contradiction again.

(ii) If $d_{l}=0$ and $c_{l} \neq 0$, according to the above analysis, we can see that the degree of the right-hand side of (8) is at most $m+(l+3) n$, if $d_{l-1} \neq 0$, then the degree of left-hand side of (8) is $2 m+(l+2) n-1$, then $n=m-1$, which is a contradiction with the assumption $m<n$; if $d_{l-1}=0$ and $d_{l-2} \neq 0$, then the degree of left-hand side of (8) is $3 m+(l+1) n-1$, then $2 n=2 m-1$, a contradiction. 
Similarly, if $d_{j}=0(j=2, \cdots, l)$, since $d_{1}=1$, we have $(l-1) n=$ $(l-1) m-1$, a contraction again.

In a word, if $\operatorname{deg}_{w}(P)=l$ and $\operatorname{deg}_{w}(Q)=l-1$, then $m \geq n$. Specially, if $c_{l}=0, c_{l-1} \neq 0$ and $d_{l} \neq 0$, we have $n=m+1$. The proof of Theorem 1 is complete.

\section{Proof of Theorem 2}

Suppose that $w(z)$ is a transcendental entire solution of (4). We consider the following equation:

$$
c(z) w(z) w(z+1)=d(z) w(z)-a(z) w^{\prime}(z)-b(z) w^{\prime \prime}(z) .
$$

If $w(z)$ is a transcendental entire solution of (9), then $w(z)$ has at most finitely many zeros.

In fact, if $w(z)$ has infinitely many multiple zeros, we can fide a zero $z_{0}$, such that

$$
d\left(z_{0}\right) w\left(z_{0}\right)-a\left(z_{0}\right) w^{\prime}\left(z_{0}\right)-b\left(z_{0}\right) w^{\prime \prime}\left(z_{0}\right) \neq 0,
$$

but the left-hand side of (9) is equal to zero at $z_{0}$, which is a contradiction.

If $w(z)$ has infinitely many single zero, by the assume that $a(z) w^{\prime}(z)+b(z) w^{\prime \prime}(z) \not \equiv 0$, we can fide a single zero $z_{1}$, such that

$$
d\left(z_{1}\right) w\left(z_{1}\right)-a\left(z_{1}\right) w^{\prime}\left(z_{1}\right)-b\left(z_{1}\right) w^{\prime \prime}\left(z_{1}\right) \neq 0,
$$

but the left-hand side of (9) is equal to zero at $z_{1}$, a contradiction again. So $w(z)$ has at most finitely many zeros. 
Thus, according to the Hadamard factorization theorem, we assume that

$$
w(z)=q(z) e^{p(z)}
$$

where $q(z)$ is non-zero polynomial and $p(z)$ is an entire function. Substituting into (9), we have

$$
\begin{aligned}
& c(z) q(z+1) e^{p(z+1)} \\
& \quad=d(z)-a(z)\left[p^{\prime}(z)+\frac{q^{\prime}(z)}{q(z)}\right]-b(z)\left[q^{\prime \prime}(z)+\left[p^{\prime}(z)\right]^{2}+\frac{q^{\prime \prime}(z)+2 p^{\prime}(z) q^{\prime}(z)}{q(z)}\right] .
\end{aligned}
$$

From (10), we can see that the order of growth of the left-hand side is always greater than the right-hand side, which is a contradiction. Therefore, (4) has no transcendental entire solutions. The proof of Theorem 2 is complete.

\section{Proof of Theorem 3}

Suppose that $w(z)$ is a transcendental meromorphic solution of (4). Rewrite (4) as

$$
c(z) w(z)=\left(d(z)-a(z) \frac{w^{\prime}(z)}{w(z)}-b(z) \frac{w^{\prime \prime}(z)}{w(z)}\right) \frac{w(z)}{w(z+1)} .
$$

Taking proximity functions from both sides of (11), and using the logarithmic derivative lemma and Lemma 3, we have

$$
m(r, w)=S(r, w) .
$$

Thus

$$
T(r, w)=N(r, w)+S(r, w) .
$$

The proof of Theorem 3 is complete. 


\section{References}

[1] W. K. Hayman, Meromorphic Functions, Oxford: Clarendon Press, 1964.

[2] Z. X. Chen and K. H. Shon, On zeros and fixed points of differences of meromorphic functions, Journal of Mathematical Analysis and Applications 344(1) (2008), 373-383.

DOI: https://doi.org/10.1016/j.jmaa.2008.02.048

[3] Z. X. Chen, Growth and zeros of meromorphic solution of some linear difference equations, Journal of Mathematical Analysis and Applications 373(1) (2011), 235-241.

DOI: https://doi.org/10.1016/j.jmaa.2010.06.049

[4] Y. M. Chiang and S. J. Feng, On the nevanlinna characteristic of $f(z+\eta)$ and difference equations in the complex plane, The Ramanujan Journal 16(1) (2008), 105-129.

DOI: https://doi.org/10.1007/s11139-007-9101-1

[5] I. Laine, J. Rieppo and H. Silvennoinen, Remarks on complex difference equations, Computational Methods and Function Theory 5(1) (2005), 77-88.

DOI: https://doi.org/10.1007/BF03321087

[6] R. G. Halburd and R. J. Korhonen, Difference analogue of the lemma on the logarithmic derivative with applications to difference equations, Journal of Mathematical Analysis and Applications 314(2) (2006), 477-487.

DOI: https://doi.org/10.1016/j.jmaa.2005.04.010

[7] R. Korhonen, A new Clunie type theorem for difference polynomials, Journal of Difference Equations and Applications 17(3) (2011), 387-400.

DOI: https://doi.org/10.1080/10236190902962244

[8] L. Y. Gao, On meromorphic solutions of a type of difference equations, Chinese Annals of Mathematics: Series A 35(2) (2014), 193-202.

[9] L. Y. Gao, Solutions of complex higher-order difference equations, Acta Mathematica Sinica 56(4) (2013), 451-458.

[10] R. Halburd and R. Korhonen, Growth of meromorphic solutions of delay differential equations, Proceedings of the American Mathematical Society 145(6) (2017), 2513-2526.

DOI: https://doi.org/10.1090/proc/13559

[11] C. J. Song, K. Liu and L. Ma, Meromorphic solutions to non-linear differentialdifference equations, Electronic Journal of Differential Equations (2018); Article 93, pp. 1-12. 
[12] I. Laine, Nevanlinna Theory and Complex Differential Equations, Walter de Gruyter, Berlin, 1993.

[13] R. Halburd, R. Korhonen and K. Tohge, Holomorphic curves with shift-invariant hyperplane preimages, Transactions of the American Mathematical Society 366(8) (2014), 4267-4298.

DOI: https://doi.org/10.1090/S0002-9947-2014-05949-7

[14] H. X. Yi and C. C. Yang, Theory of the Uniqueness of Meromorphic Functions (in Chinese), Beijing: Science Press, 1995. 